\title{
Back from the dead: TIL apoptosis in cancer immune evasion
}

\author{
Brendan L Horton ${ }^{1}$ and Thomas F Gajewski ${ }^{\star}{ }^{1}$ \\ ${ }^{1}$ Department of Pathology, University of Chicago, Chicago, IL, USA
}

Tumours from multiple cancer types can be infiltrated by $\mathrm{CD} 8{ }^{+} \mathrm{T}$ cells (TILs) (Spranger et al, 2016). TILs are thought to be suppressed by multiple immune inhibitory molecules in the tumour microenvironment, and this suppression has been associated with tumour progression (Gajewski et al, 2013). Therefore, despite tumour infiltration, almost all tumours containing TILs will progress if not treated. While several immune inhibitory mechanisms have been identified, immune inhibitory receptors expressed on activated T cells, like CTLA-4 and PD-1, have received the most attention over recent years owing to the immense clinical success of PD-1 and CTLA-4 neutralising antibodies (Hodi et al, 2010; Topalian et al, 2012). The engagement of inhibitory receptors expressed by TILs is thought to render TILs dysfunctional. However, evidence from both human tumour samples and mouse models has suggested that, despite inhibitory receptor expression, TILs are not functionally inert and actually retain the ability to proliferate, produce IFN- $\gamma$ (Daud et al, 2016; Williams et al, 2017), and show ex vivo cytotoxicity (Mahnke et al, 2012). These observations raise the question of why activated TILs are not able to spontaneously control progressing tumours, and how tumours that contain TILs might sometimes be resistant to immunotherapies such as checkpoint blockade. Current immunotherapies can induce durable tumour regression; however, they benefit a minority of patients: finding new strategies to increase the response rate to immunotherapies is of great interest to both researchers and clinicians.

Two new studies have reported that $\mathrm{CD}^{+}$TILs undergo apoptosis within the tumour microenvironment, indicating that their apoptosis is a contributing factor to the frequent failure of TILs to reject tumours. Studies from Zhu et al (Zhu et al, 2017) and from our own laboratory (Horton et al, 2017) found that antigenspecific $\mathrm{CD}^{+} \mathrm{T}$ cells undergo apoptosis in the tumour microenvironment, limiting their ability to control progressing tumours and respond to immunotherapy. Zhu et al utilised an inducible melanoma model expressing a tumour antigen, P1A, to test for responsiveness to various types of immunotherapy. They found that adoptive cell transfer (ACT) of activated CD8 ${ }^{+} \mathrm{T}$ cells into the murine model was able to regress transplanted melanomas, but not induced melanomas. From this finding, the authors investigated the differences between the transplanted and induced melanoma models. The authors found that in induced melanomas, where ACT was unsuccessful, both the transferred and endogenous $\mathrm{CD} 8{ }^{+}$TILs underwent increased apoptosis compared with the transplanted tumours. In their model, TIL apoptosis was dependent on IFN- $\gamma$, which increased at the tumour site after ACT. Increased IFN- $\gamma$ led to induction of the apoptosis initiator FasL in the tumour microenvironment, particularly on myeloid-derived suppressor cells (MDSCs) which were enriched in the induced tumours. Blockade of FasL-Fas interactions with an Fas-Fc decreased TIL apoptosis, as did depletion of MDSCs. Furthermore, blockade of FasL-Fas signalling and MDSC depletion synergised with checkpoint blockade to slow the growth of induced melanomas (Figure 1).

The study by Horton and colleagues also found that apoptosis of $\mathrm{CD}^{+}$TILs limited endogenous anti-tumour immunity, in this case utilising the transplantable B16.SIY melanoma model, a B16 melanoma line that was engineered to express the $\mathrm{CD}^{+}{ }^{+} \mathrm{T}$-cell antigen SIY. They tracked antigen-specific TILs using either $\mathrm{K}^{\mathrm{b}}$ / SIY pentamers or surface expression of both LAG-3 and 4-1BB, a phenotype which was previously found to identify dysfunctional, tumour antigen-specific cells in the tumour microenvironment (Williams et al, 2017). The authors found that LAG-3 ${ }^{+} 4-1 \mathrm{BB}^{+}$ $\mathrm{CD}^{+}$TILs constantly proliferated in situ; however, despite this constant proliferation there was no accumulation of antigenspecific $\mathrm{CD} 8{ }^{+}$TILs as the tumours progressed. Investigating this phenomenon, Horton and colleagues found that antigen-specific $\mathrm{CD}^{+}$TILs underwent apoptosis in the steady state during tumour progression, indicating that a futile cycle of proliferation and apoptosis was linked to T-cell dysfunction in the tumour microenvironment. Utilising multiple transplantable cell lines as well as an autochthonous, inducible melanoma model, they determined that apoptosis of antigen-specific TILs occurred in progressing, but not spontaneously regressing, tumours. This reduction in TIL apoptosis in regressing tumours is also consistent with observations by Zhu and colleagues, who found reduced TIL apoptosis during tumour regression induced by ACT. In their own 


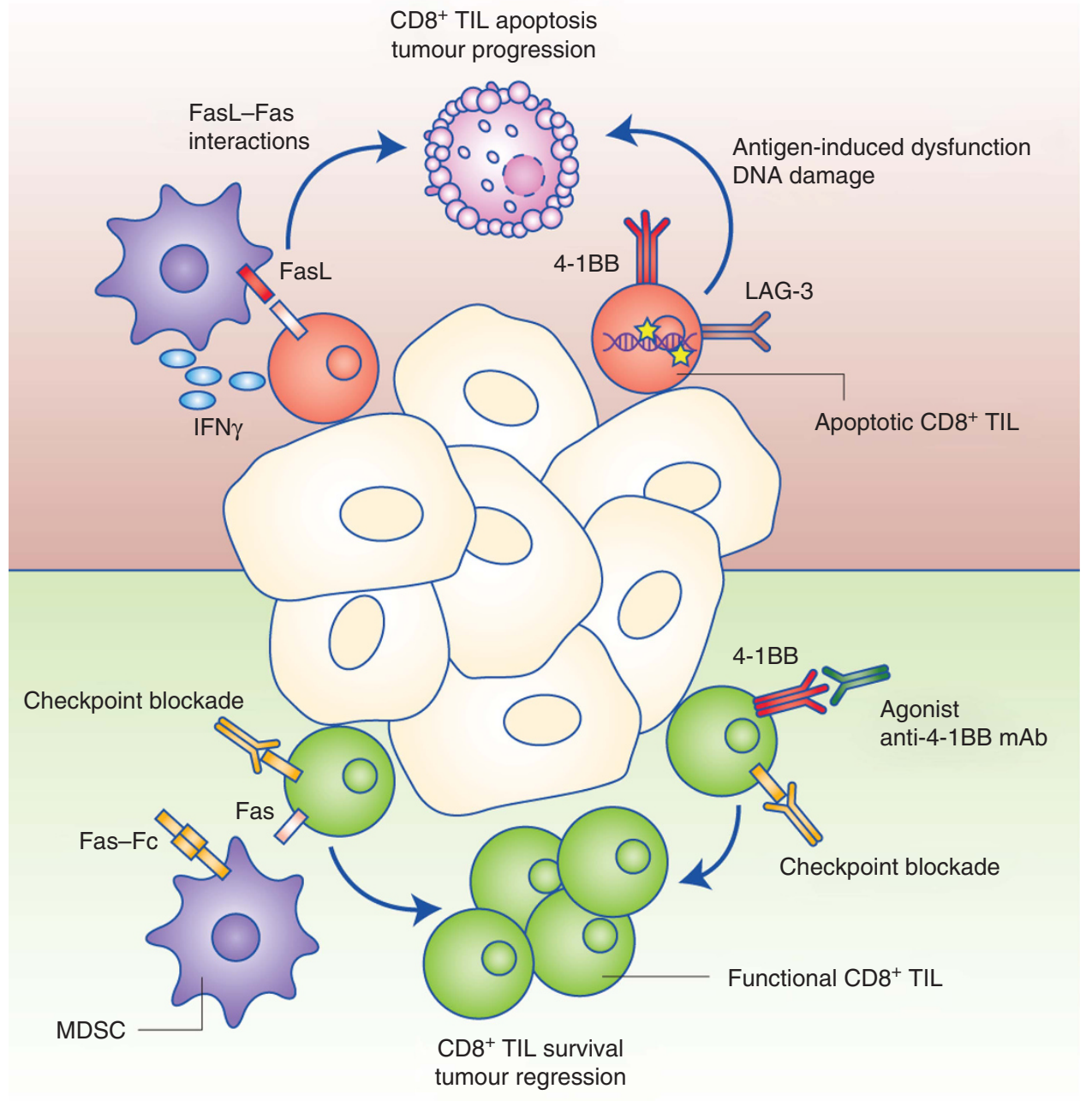

Figure 1. Tumour antigen-specific TILs undergo apoptosis during tumour progression (top): Zhu and colleagues found that TIL apoptosis was a result of FasL expression on MDSCs that was driven by TIL-produced IFN $\gamma$. Horton and colleagues found that TIL apoptosis was associated with antigen-induced T-cell dysfunction, and possibly due to the accumulation of DNA damage in dysfunctional TILs. Inhibiting TIL apoptosis enhances tumour control (bottom): Zhu and colleagues found that administration of an Fas-Fc protein and depletion of MDSCs enhanced the efficacy of checkpoint blockade. Horton and colleagues found that an agonist 4-1BB antibody synergised with checkpoint blockade to decrease TIL apoptosis and enhance tumour control. Mechanisms of human TIL apoptosis should be investigated in order to develop therapeutic strategies to enhance TIL survival.

ACT experiments, Horton and colleagues found that overexpression of $\mathrm{Bcl}-\mathrm{x}_{\mathrm{L}}$ in transferred antigen-specific $\mathrm{T}$ cells increased tumour control, clearly demonstrating that apoptosis was a significant barrier to T-cell-mediated tumour rejection.

In studying the mechanism of TIL apoptosis, Horton and colleagues found that the proliferating/apoptosing $\mathrm{CD}^{+}{ }^{+} \mathrm{T}$ cells showed DNA damage response induction by gene expression profiling, and revealed evidence for DNA damage by staining for $\gamma \mathrm{H} 2 \mathrm{AX}$. Interestingly, DNA damage was not found in proliferative $\mathrm{T}$ cells in spleens or lymph nodes, indicating that this phenomenon was restricted to proliferating $\mathrm{T}$ cells within the tumour site. The results implied that $\mathrm{T}$-cell activation and proliferation, specifically in the tumour microenvironment, leads to DNA damage and apoptosis. These results raise the possibility that oxidative stress from the noxious tumour environment could lead to the accumulation of DNA damage that results in TIL apoptosis.

Results from these studies highlight that apoptosis of TILs may be a critical part of the failure of the immune system to reject tumours, both during steady state and during immunotherapy. In both studies, modulating apoptosis increased tumour control by checkpoint blockade. Zhu and colleagues found that inhibiting FasL-Fas signalling and depleting MDSCs increased the responsiveness to checkpoint blockade in the inducible melanoma model, whereas Horton and colleagues determined that the synergy between checkpoint blockade and agonist anti-4-1BB antibodies corresponded to a large decrease in the apoptosis of antigenspecific TILs. The subtle differences between models could be related to the tumour type, the composition of immune suppressive cells within the tumour microenvironment, and the strength of antigen stimulation. Nevertheless, it will be critical to determine the role TIL apoptosis plays in human tumours, as therapeutic strategies could be developed to overcome this barrier.

\section{CONFLICT OF INTEREST}

The authors declare no conflict of interest. 


\section{REFERENCES}

Daud AI, Loo K, Pauli ML, Sanchez-Rodriguez R, Sandoval PM, Taravati K, Tsai K, Nosrati A, Nardo L, Alvarado MD, Algazi AP, Pampaloni MH, Lobach IV, Hwang J, Pierce RH, Gratz IK, Krummel MF, Rosenblum MD (2016) Tumor immune profiling predicts response to anti-PD-1 therapy in human melanoma. J Clin Invest 126: 3447-3452.

Gajewski TF, Schreiber H, Fu YX (2013) Innate and adaptive immune cells in the tumor microenvironment. Nat Immunol 14: 1014-1022.

Hodi FS, O'Day SJ, McDermott DF, Weber RW, Sosman JA, Haanen JB, Gonzalez R, Robert C, Schadendorf D, Hassel JC, Akerley W, van den Eertwegh AJ, Lutzky J, Lorigan P, Vaubel JM, Linette GP, Hogg D, Ottensmeier CH, Lebbé C, Peschel C, Quirt I, Clark JI, Wolchok JD, Weber JS, Tian J, Yellin MJ, Nichol GM, Hoos A, Urba WJ (2010) Improved survival with ipilimumab in patients with metastatic melanoma. $N$ Engl J Med 363: 711-723.

Horton BL, Williams JB, Cabanov A, Spranger S, Gajewski TF (2017) Intratumoral CD8 + T-cell apoptosis is a major component of T cell dysfunction and impedes anti-tumor immunity. Cancer Immunol Res 6: 1-11.

Mahnke YD, Devevre E, Baumgaertner P, Matter M, Rufer N, Romero P, Speiser DE (2012) Human melanoma-specific CD8(+) T-cells from metastases are capable of antigen-specific degranulation and cytolysis directly ex vivo. Oncoimmunology 1: 467-530.

Spranger S, Luke JJ, Bao R, Zha Y, Hernandez KM, Li Y, Gajewski AP, Andrade J, Gajewski TF (2016) Density of immunogenic antigens does not explain the presence or absence of the T-cell-inflamed tumor microenvironment in melanoma. Proc Natl Acad Sci USA 113: E7759-E7768.

Topalian SL, Hodi FS, Brahmer JR, Gettinger SN, Smith DC, McDermott DF, Powderly JD, Carvajal RD, Sosman JA, Atkins MB, Leming PD, Spigel DR, Antonia SJ, Horn L, Drake CG, Pardoll DM, Chen L, Sharfman WH, Anders RA, Taube JM, McMiller TL, Xu H, Korman AJ, Jure-Kunkel M, Agrawal S, McDonald D, Kollia GD, Gupta A, Wigginton JM, Sznol M (2012) Safety, activity, and immune correlates of anti-PD-1 antibody in cancer. N Engl J Med 366: 2443-2454.

Williams JB, Horton BL, Zheng Y, Duan Y, Powell JD, Gajewski TF (2017) The EGR2 targets LAG-3 and 4-1BB describe and regulate dysfunctional antigen-specific CD8 $+\mathrm{T}$ cells in the tumor microenvironment. J Exp Med 214: 381-400.

Zhu J, Powis de Tenbossche CG, Cané S, Colau D, van Baren N, Lurquin C, Schmitt-Verhulst AM, Liljeström P, Uyttenhove C, Van den Eynde BJ (2017) Resistance to cancer immunotherapy mediated by apoptosis of tumor-infiltrating lymphocytes. Nat Commun 8: 1404. 